\title{
Two-Dimensional X-ray Photoelectron Spectroscopy for Composite Surface Analysis
}

\author{
Sefik Suzer, ${ }^{*, \dagger}$ Hikmet Sezen, ${ }^{\dagger}$ and Aykutlu Dâna*,* \\ Departments of Chemistry and Physics and Institute of Materials and Nanotechnology, Bilkent University, \\ 06800 Ankara, Turkey
}

We describe a method for obtaining two-dimensional X-ray photoelectron spectroscopic data derived from the frequency dependence of the XPS peaks recorded under electrical square-wave pulses, which control and affect the binding energy positions via the electrical potentials developed as a result of charging. By using cross-correlations between various peaks, our technique enables us to elucidate electrical characteristics of surface structures of composite samples and bring out various correlations between hidden/overlapping peaks.

X-ray photoelectron spectroscopy (XPS, ESCA, or PES) has been employed widely as a vital analysis tool, by all researchers in natural and applied sciences, during the last 5 decades. The technique, using conventional ${ }^{1}$ and later-on monochromatic Xrays, ${ }^{2}$ was introduced by Kai Siegbahn and his co-workers for which Prof. Siegbahn was rewarded with the Nobel Prize in 1981. The technique has flourished tremendously through utilization of synchrotron radiation, ${ }^{3}$ and most important developments in chemical, physical, molecular, material, catalysis, semiconductor,

* Corresponding authors. E-mail: suzer@fen.bilkent.edu.tr (S.S.) and aykutlu@ fen.bilkent.edu.tr (A.D.).

${ }^{\dagger}$ Department of Chemistry and Institute of Materials and Nanotechnology.

* Department of Physics and Institute of Materials and Nanotechnology.

(1) Siegbahn, K.; Hammond, D.; Fellner-Feldegg, H.; Barnett, E. F. Science 1972, 176, 245.

(2) Siegbahn, K. Science 1982, 217, 111.

(3) Smith, N. V.; Woodruff, D. P. Science 1982, 216, 367.

(4) (a) Maier, F.; Gottfried, J. M.; Rossa, J.; Gerhard, D.; Schultz, P. S.; Schwinger, W.; Wasserscheid, P.; Steinruck, H. P. Angew. Chem., Int. Ed. 2006, 45, 7778. (b) Chaudhury, M. K.; Whitesides, G. M. Science 1992, 255, 1230. (c) Steinberg, S.; Ducker, W.; Vigil, G.; Hyukjin, C.; Frank, C.; Tseng, M. Z.; Clarke, D. R.; Israelachvili, J. N. Science 1993, 260, 656. (d) Gref, R.; Minamitake, Y.; Peracchia, M. T.; Trubetskoy, V.; Torchilin, V.; Langer, R. Science 1994, 263, 1600. (e) Schierbaum, K. D.; Weiss, T.; Vanvelzen, E. U. T.; Engbersen, J. F. J.; Reinhoudt, D. N.; Gopel, W. Science 1994, 265, 1413. (f) Kay, A.; Arenholz, E.; Mun, S.; de Abajo, F. J. G.; Fadley, C. S.; Denecke, R.; Hussain, Z.; Van Hove, M. A. Science 1998, 281, 679. (g) Valden, M.; Lai, X.; Goodman, D. W. Science 1998, 281, 1647. (h) Asahi, R.; Morikawa, T.; Ohwaki, T.; Aoki, K.; Taga, Y. Science 2001, 293, 269. (i) McKee, R. A.; Walker, F. J.; Nardelli, M. B.; Shelton, W. A.; Stocks, G. M. Science 2003, 300, 1726. (j) Kim, H. J.; Graham, D. W.; DiSpirito, A. A.; Alterman, M. A.; Galeva, N.; Larive, C. K.; Asunskis, D.; Sherwood, P. M. A. Science 2004, 305, 1612. (k) Ghosal, S.; Hemminger, J. C.; Bluhm, H.; Mun, B. S.; Hebenstreit, E. L. D.; Ketteler, G.; Ogletree, D. F.; Requejo, F. G.; Salmeron, M. Science 2005, 307, 563. (l) Tulevski, G. S.; Myers, M. B.; Hybertsen, M. S.; Steigerwald, M. L.; Nuckolls, C. Science 2005, 309, 591. biomedical, and nano science and technologies have, one way or another, referred to the results of this pivotal surface analytical tool. ${ }^{4}$ Analytical applications of photoelectron spectroscopy have also been reviewed frequently. ${ }^{5}$

Almost exclusively, the technique to date has been utilized in a static, data-gathering mode, concentrating mostly on recording line positions and intensities, and in a few cases, diffraction methods ${ }^{6}$ have been incorporated, but attempts for recording XPS data for obtaining dynamical information as a response to a stimuli have been scarce. On the other hand, materials respond to external stimuli, like optical, electrical, magnetic, thermal, chemical, mechanical, etc., and their responses, properly recorded, give important information about the system(s) under investigation. This is particularly important for core-level XPS measurements, since these responses can be followed with chemical specificity. ${ }^{7}$ Several reports have appeared on recording the responses of systems by XPS, under only optical stimuli (mostly by one or two photon laser excitation or ionization). ${ }^{8}$ Our group has been reporting studies on probing response(s) using core-level photoelectron measurements, under electrical stimuli in the form of square-wave pulses. ${ }^{9}$ In the present contribution, we extend our studies to even more detailed analysis of these responses using

(5) Turner, N. H.; Schreifels, J. A. Anal. Chem. 2000, 70, 99R. (and the references therein).

(6) (a) Barton, J. J. Phys. Rev. Lett. 1988, 61, 1356. (b) Fadley, C. S. Prog. Surf. Sci. 1984, 16, 275.

(7) (a) Doron-Mor, H.; Hatzor, A.; Vaskevich, A.; van der Boom-Moav, T.; Shanzer, A.; Rubinstein, I.; Cohen, H. Nature 2000, 406, 382. (c) Shabtai, K.; Rubinstein, I.; Cohen, S. R.; Cohen, H. J. Am. Chem. Soc. 2000, 122, 4959. (d) Cohen, H. Appl. Phys. Lett. 2004, 85, 1271.

(8) (a) Bauer, M.; Lei, M. C.; Read, K.; Tobey, R.; Gland, J.; Murnane, M. M.; Kapteyn, H. C. Phys. Rev. Lett. 2001, 87, 025501. (b) Bjorneholm, O.; Nilsson, A.; Sandell, A.; Hernnas, B.; Martensson, N. Phys. Rev. Lett. 1992, 68, 1892. (c) Drescher, M.; Hentschel, M.; Kienberger, R.; Uiberacker, M.; Yakovlev, V.; Scrinizi, A.; Westerwalbesloh, T.; Kleineberg, U.; Heinzmann, U.; Krausz, F. Nature 2002, 419, 803. (d) Hofer, U.; Shumay, I. L.; Reuss, C.; Thomann, U.; Wallauer, W.; Fauster, T. Science 1997, 277, 1480. (e) Hovel, H.; Barke, I.; Boyen, H. G.; Ziemann, P.; Garnier, M. G.; Oelhafen, P. Phys. Rev. B 2004, 70, 5. (f) Hovel, H.; Grimm, B.; Pollmann, M.; Reihl, B. Phys. Rev. Lett. 1998, 81, 4608. (g) Marsi, M. M.; Belkhou, R.; Grupp, C.; Panaccione, G.; Taleb-Ibrahimi, A.; Nahon, L.; Garzella, D.; Nutarelli, D.; Renault, E.; Roux, R.; Couprie, M. E.; Billardon, M. Phys. Rev. B 2000, 61, R5070.

(9) (a) Demirok, U. K.; Ertas, G.; Suzer, S. J. Phys. Chem. B 2004, 108, 5179. (b) Ertas, G.; Demirok, U. K.; Atalar, A.; Suzer, S. Appl. Phys. Lett. 2005, 86, 183110. (c) Karabudak, E.; Demirok, U. K.; Suzer, S. Surf. Sci. 2006, 600, L12. (d) Sezen, H.; Ertas, G.; Dana, A.; Suzer, S. Macromolecules 2007, 40,4109 . 


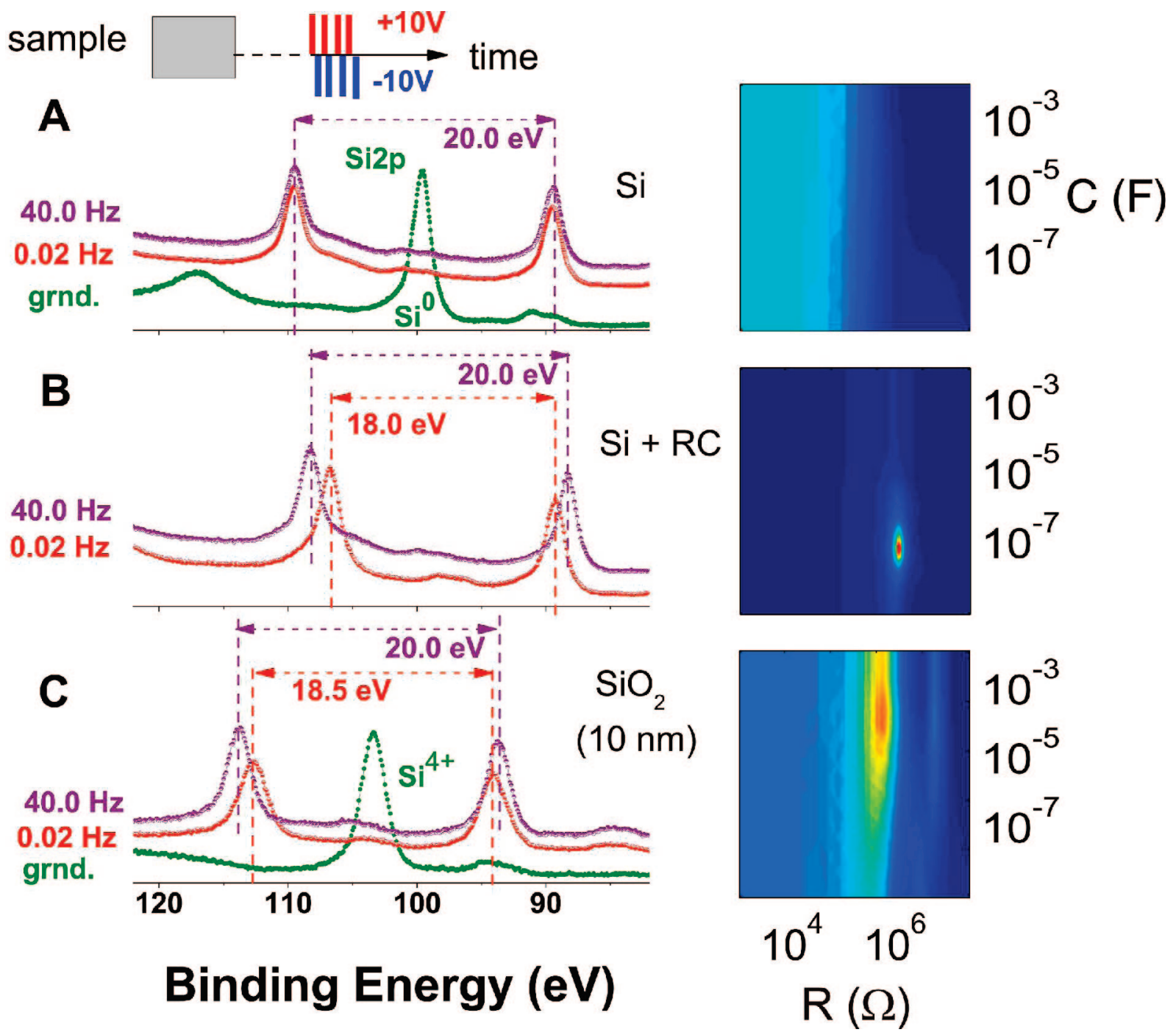

Figure 1. (A) Si2p region of the XPS spectrum of a clean and conducting silicon $\left(\mathrm{Si}^{\circ}\right)$ sample recorded when grounded and under 40.0 and $0.02 \mathrm{~Hz} \pm 10 \mathrm{~V}$ square wave excitation, which causes the peaks to double at -10 and $+10 \mathrm{eV}$ positions, respectively, at all frequencies. (B) The same spectrum of the clean Si sample, this time connected through an external $R C(1 \mathrm{M} \Omega, 56 \mathrm{nF})$ circuit. The peaks are also doubled but, as a result of charging through the $R C$ circuit, the peaks do not appear at $\pm 10 \mathrm{eV}$ positions at low frequencies. (C) Spectrum of a less conducting sample containing approximately $10 \mathrm{~nm}$ silicon oxide layer $\left(\mathrm{Si}^{4+}\right)$. Here, the peak separation becomes smaller than $20 \mathrm{eV}$ at low frequencies, due to charging of the oxide layer. The right-hand side of the figure shows the inverse of the resulting mean-square-error MSE ${ }^{-1}(R, C)$ as two-dimensional contour plots, where locations with perfect fits (or lowest errors) appear as bright spots, for clean silicon, the $10 \mathrm{~nm}$ silicon dioxide, and the artificially created systems consisting of the clean silicon connected through and external $R C$ circuit (1 M $\Omega, 56 \mathrm{nF}$ ). Blue indicates a low and red indicates a high probability. Note that our method correctly predicts a very localized contour $(\sim 1 \mathrm{M} \Omega$ and $\sim 56 \mathrm{nF})$ for the artificial dielectric as the sign of reliability of our technique.

correlation techniques. This approach results in creating a novel two-dimensional X-ray photoelectron spectroscopy (2D-XPS), similar to two-dimensional nuclear magnetic resonance (2DNMR), ${ }^{10}$ and/or two-dimensional IR spectroscopies. ${ }^{11}$ As we will demonstrate below, this simple extension enables us to extract various hidden information related with dielectric properties of composite surface structures with chemical specificity.

The technique is based on the control of low-energy neutralizing electron current incident on the sample by an external bias applied, and thereby control of the electrical potential developed via differential charging, which manifests and can easily be probed

(10) Meier, B. H.; Ernst, R. R. J. Am. Chem. Soc. 1979, 101, 6441.

(11) Zheng, J. R.; Kwak, K.; Ashbury, J.; Chen, X.; Piletic, I. R.; Fayer, M. D. Science 2005, 309, 1338. as shifts in the binding energy positions of the XPS peaks, was also extensively reported by others. ${ }^{12}$ As the frequency of the applied bias is changed, the response of the system also changes due to its finite resistance and capacitance, as illustrated in Figure 1. Moreover, different surface structures and/or domains display different charging behavior due to their intrinsic electrical properties as in the case of an approximately $10 \mathrm{~nm}$ thermal $\mathrm{SiO}_{2}$ layer also shown in Figure 1C. Interestingly, the conducting silicon sample behaves like a dielectric after connecting it through an external $R C$ circuit as displayed in Figure 1B.

(12) (a) Lau, W. M. J. Appl. Phys. 1989, 65, 2047. (b) Lau, W. M.; Wu, X. W. Surf. Sci. 1991, 245, 345. (c) Sherwood, P. M. A. Surf. Sci. 2006, 600, 771. (d) Gouzman, I.; Dubey, M.; Carolus, M. D.; Schwartz, J.; Bernasek, S. L. Surf. Sci. 2006, 600, 773. (e) Dubey, M.; Gouzman, I.; Bernasek, S. L.; Schwartz, J. Langmuir 2006, 22, 4649. 

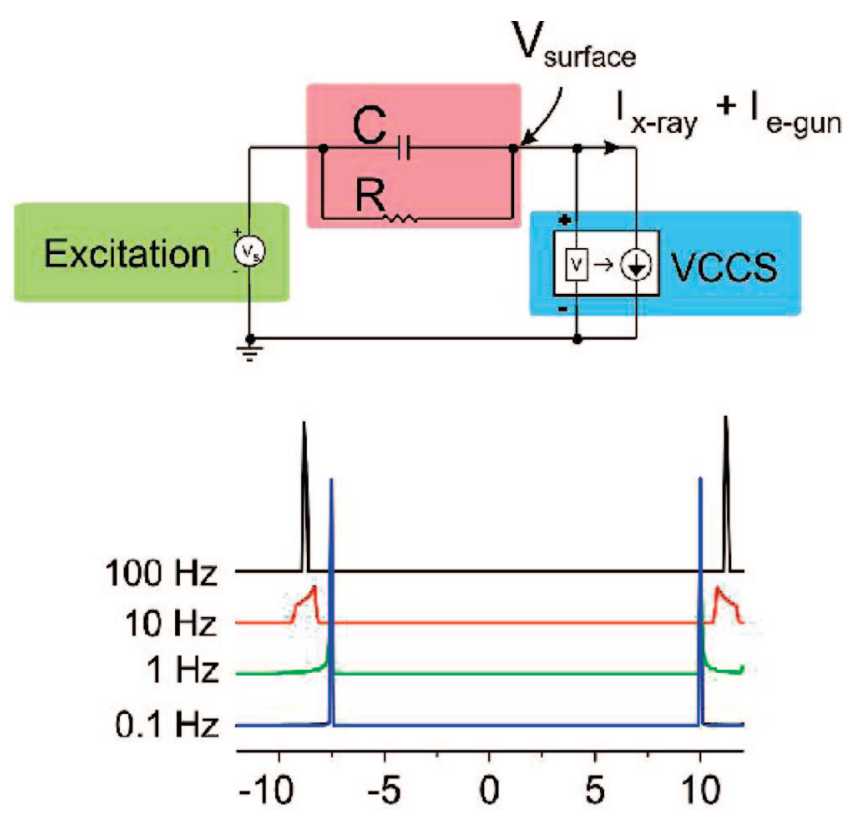

Figure 2. The circuit used for simulation of the frequency dependence and the line-shapes calculated for a square wave excitation for $1 \mathrm{M} \Omega$ and $56 \mathrm{nF}$.

As we had already reported, our measurements can be modeled with a lumped circuit as shown in Figure 2, where the electron gun and the photoelectron currents are modeled by a voltagecontrolled currentsource(VCCS). Thenonlinearvoltage-current curve for the VCCS was extracted through analysis of a series of XPS measurements by applying dc voltages to the clean silicon sample in series with a known external resistor. The VCCS current-voltage dependence is assumed to be the same throughout the entire dynamic measurements. The excitation voltage source $V_{\mathrm{EX}}(t)$ can be a time varying voltage with arbitrary time dependence. In order to calculate the XPS spectra, we need to solve the differential equation describing the change of the surface potential $V_{\mathrm{S}}(t)$ given by

$$
C \frac{\mathrm{d}\left(V_{\mathrm{S}}-V_{\mathrm{EX}}\right)}{\mathrm{d} t}+\frac{V_{\mathrm{S}}-V_{\mathrm{EX}}}{R}+I_{\mathrm{s}}\left(V_{\mathrm{S}}\right)=0
$$

where, $R$ and $C$ are, respectively, the effective resistance and the capacitance between the surface and the source as shown in Figure 2, and $I_{\mathrm{S}}\left(V_{\mathrm{S}}\right)$ is the surface potential dependent current of the electron flood-gun. Solutions can be obtained by numerical integration for arbitrary excitations $V_{\mathrm{EX}}(t)$. For a periodic excitation of period $T$, one can calculate a line-shape function that gives the intensity of the XPS signal at a voltage shift of $v$ as

$$
g(v)=\int_{0}^{T} \delta\left[V_{\mathrm{S}}(t)-v\right] \mathrm{d} t
$$

Note that $\int_{-\infty}^{\infty} g(v) \mathrm{d} v=1$ and hence the line-shape function is properly normalized. It can be seen that, depending only on the period of the excitation and charge-discharge time constants of the sample, the left and right peaks of the line-shape function shift differently. If the excitation signal period is much higher than the sample time constants, we are working in a regime similar to dc. In this case, the peaks are shifted almost to the positions that would be obtained by applying -10 or $+10 \mathrm{~V}$ (dc) to the sample, respectively, and the broadening is almost zero. It is seen through calculations that, for the square-wave case, the peaks have maximum broadening as half of the period of excitation coincides with the charging-discharging time constant of the sample.

The XPS spectrum recorded by the spectrometer $S_{\mathrm{XPS}}(v)$ can now be simulated by convolution of the line-shape function $g(v)$ with the original spectrum $S_{0}(v)$ obtained by grounding the sample.

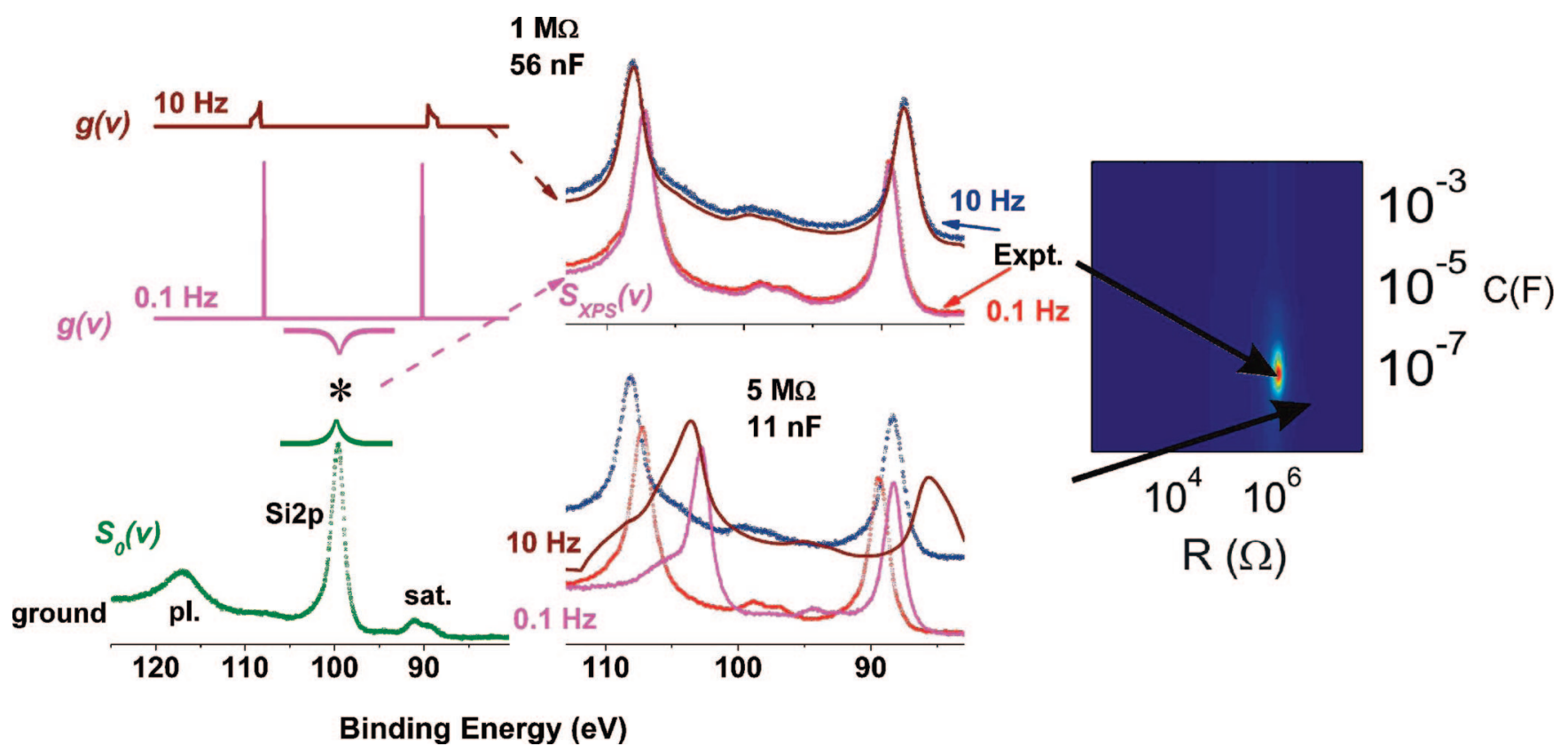

Figure 3. XPS spectrum (olive) of clean Si recorded when the sample is grounded $S_{0}(v)$. Convolution of this spectrum with the calculated line functions $g(v)$ at 0.01 (magneta) and $10 \mathrm{~Hz}$ (wine) and $R=1 \mathrm{M} \Omega$ and $C=56 \mathrm{nF}$ are also given together with the experimental spectra (red and blue). Simulated spectra for the same frequencies but using $R=11 \mathrm{M} \Omega$ and $C=5 \mathrm{nF}$ are also given which are widely different from the experimental data. The right-hand-side of the figure is the same $R C$ plot as in Figure 1 for the Si sample connected through an external $R C$ ( 1 $\mathrm{M} \Omega, 56 \mathrm{nF}$ ) circuit. 


$$
S_{\mathrm{XPS}}(v)=\int_{-\infty}^{\infty} g(v-\lambda) \mathrm{d} \lambda=g(v) S_{0}(v)
$$

As an example, we show in Figure 3 the spectra simulated using eq 3 for the values of $R=1 \mathrm{M} \Omega$ and $C=56 \mathrm{nF}$ for the square-wave excitation and compare the results with the experimentally measured spectra at frequencies of 0.01 and $10 \mathrm{~Hz}$ respectively, where the fit is almost perfect. In the same figure we also show the same for the case of $R=5 \mathrm{M} \Omega$ and $C=11 \mathrm{nF}$ where the fit to the experimental data is very far from perfect.

The model can then be used to calculate the mean-square error MSE $(R, C)$, between the convoluted and actual XPS data for a given pulse frequency, a given $R, C$, and a binding energy by selecting windows of arbitrary width (about $1-2 \mathrm{eV}$ ) centered around the positive and negative up peaks of the lineshape functions shifted with the desired energy. Summing the mean-square-errors for multiple frequencies (in this case six), we get an overall mean-square-error that gives us a measure of how good the given $R$ and $C$ values explain the frequency dependence of the part of the XPS spectrum around a given binding energy. When the inverse of the resulting mean-squareerror $\mathrm{MSE}^{-1}(R, C)$ is plotted, the best fit of the electrical parameters of the system $(R$ and $C$ ) can be inferred. If we plot the inverse of the mean-square-errors in a two-dimensional plot,
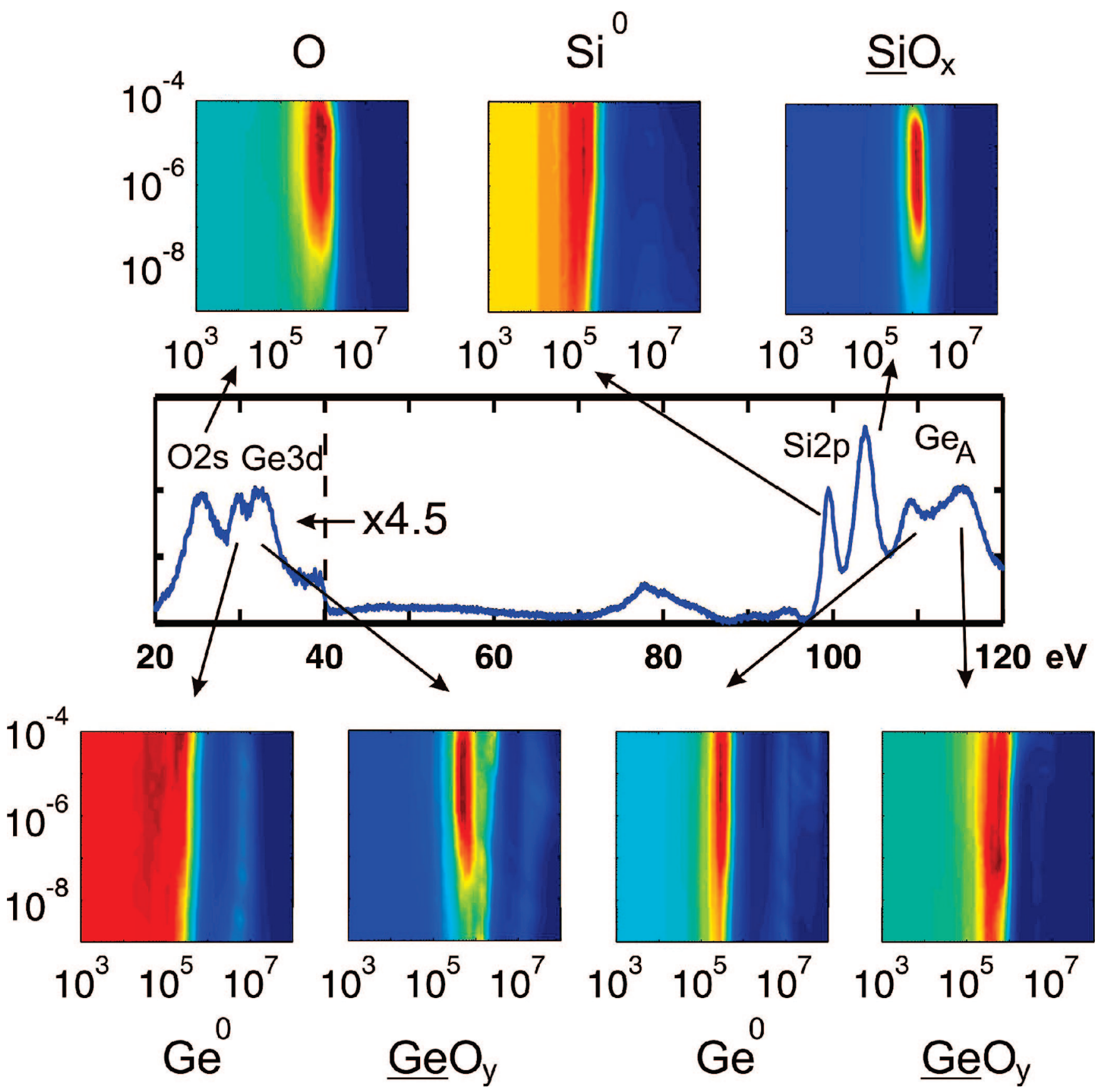

Figure 4. 20-120 eV region of the XPS spectrum and the derived electrical parameters for a composite sample consisting of germanium nanoclusters prepared within a silicon oxide matrix grown on silicon for use as flash-memory devices. ${ }^{9}$ Each peak or region can be analyzed separately in terms of its $R$ and $C$ components. Note that similarities and also differences between various peaks or regions are very clear to establish. 


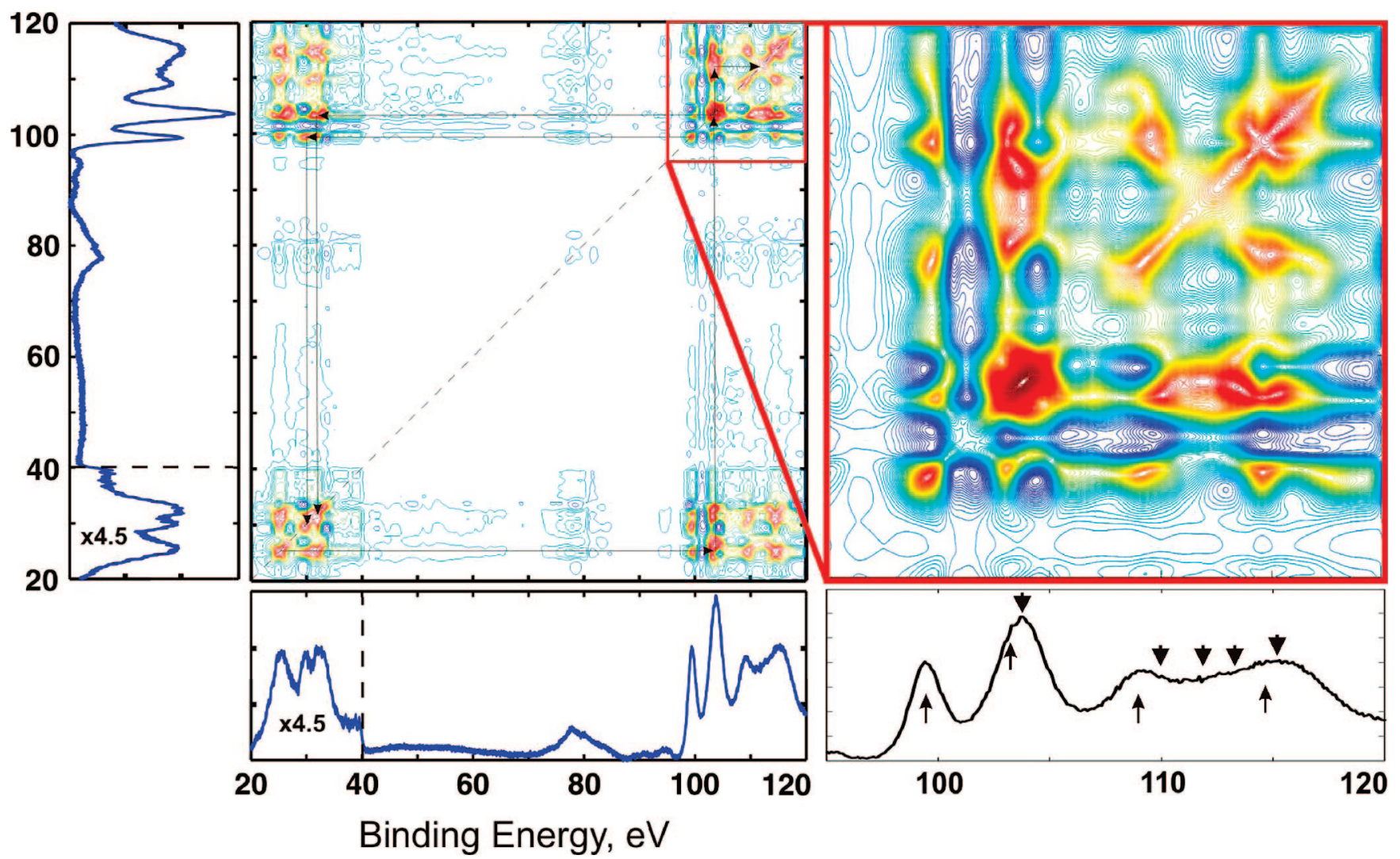

Figure 5. 2-D XPS correlation plot for the spectrum shown in Figure 2. The autocorrelations are in the diagonal, and the cross-correlations between different peaks are given in the off-diagonals. Here again, blue and red indicate low and high correlations, respectively. In addition to clear cross-correlations between various peaks and/or regions, several hidden peaks emerge in the unresolved region between 110-120 eV, three of which correlate with the $\mathrm{Si}^{\circ}$ and four with the $\mathrm{Si}^{4+}$ peaks as designated in the lower part of the figure.

locations with perfect fits (or lowest errors) appear as bright spots in the $R C$ fit plots.

The results of this procedure are also shown in the right-handside of the Figure 1, for the clean $\mathrm{Si}$, the $10 \mathrm{~nm} \mathrm{SiO}_{2}$ thermal oxide-layer, and the artificially created dielectric system consisting of the clean $\mathrm{Si}$ connected externally through the $R C$ circuit $(1$ $\mathrm{M} \Omega, 56 \mathrm{nF}$ ). The model correctly predicts the $R$ and the $C$ of the artificial system, predicts a very low $R$ and a very low $C$ for the clean $\mathrm{Si}$, and predicts $\sim 1 \mathrm{M} \Omega$ resistance and a wide range of $C \mathrm{~s}$ for the $\mathrm{SiO}_{2}$ system, most probably due to the multitude of defects within the thermal oxide layer and/or defects created by the X-rays.

We have applied the technique for analysis of a more complex and composite sample consisting of germanium nanoclusters prepared within a silicon oxide matrix grown on silicon for potential use as flash-memory devices., ${ }^{14}$ Annealing the sample after preparation yields germanium nanoparticles, $\mathrm{Ge}(\mathrm{np})$, within the silicon oxide matrix. The spectrum, shown in Figure 4, corresponds to the part of the XPS data with the O2s $(\sim 24 \mathrm{eV})$, Ge3d $(\sim 28 \mathrm{eV})$, Si2p $\left(100 \mathrm{eV}\right.$ for $\mathrm{Si}^{0}$ and $\sim 104 \mathrm{eV}$ for the $\left.\mathrm{Si}^{4+}\right)$, and the $\mathrm{Ge}_{\text {LMM }}(\sim 110-120 \mathrm{eV})$ Auger peaks. Information content of this figure is very rich. The Si2p of the substrate $\left(\mathrm{Si}^{0}\right)$, as in Figure 1, has very low $R$ and $C$ values, whereas the Si2p of the oxide $\mathrm{SiOx}$ has again $\sim 1 \mathrm{M} \Omega$ and a wide range of $C$ values. Similarly, there are two chemically distinct Ge species, evidenced

(13) Suzer, S.; Dana, A. J. Phys. Chem. B 2006, 110, 19112.

(14) Foss, S.; Finstad, T. G.; Dana, A.; Aydinli, A. Thin Solid Films 2007, 515, 6381. both from the Ge3d and also from the Ge Auger regions. One of the Ge species has low $R$ and $C$ values which can be assigned to the germanium nanoclusters, $\mathrm{Ge}^{0}(\mathrm{np})$, and the other has a range of $R$ and $C$ values, smaller than that of the $\mathrm{SiO}_{\mathrm{x}}$ peak and can be assigned to the oxide layer around $\mathrm{GeO}_{\mathrm{y}}$. Interestingly, the $\mathrm{O} 2 \mathrm{~s}$ is almost a mixture of $\mathrm{SiO}_{\mathrm{x}}$ and the $\mathrm{GeO}_{\mathrm{y}}$ peaks, hinting that the oxide layer of the germanium nanoclusters are electrically different from those of the silicon oxide matrix they reside in, which will be brought out more clearly after analyzing their correlation data.

A one-to-one comparison of the $R$ and $C$ values we derive from our measurements with those estimated from the known resistivity and dielectric constant of the bulk silicon dioxide should not be expected, since the XPS derived values are related with trapping and detrapping of the holes created in the valence band of the oxide after the very fast $\left(10^{-12} \mathrm{~s}\right)$ photoemission process and are derived under X-ray exposure. ${ }^{15}$ The analytical power of the technique is much more important than the electrical parameters derived. In any event, the product of $R C$, i.e., the time constants, we measure for the silicon dioxide layer is comparable to those derived from time-dependent leakage currents determined for MOS systems under X-ray exposure ${ }^{16}$ and/or using scanning capacitance microscopy. ${ }^{17}$

Extending the model to create 2-D correlation plots requires a few additional computational steps. The mean-square error matrics $M_{i}(R, C)$ are calculated as described above for each binding energy $E_{i}$. The matrics are then normalized using

(15) (a) Iwata, S.; Ishizaka, A. J. Appl. Phys. 1996, 79, 6653. (b) Cazaux; J. J. Electron Spectrosc. Relat. Phenom. 1999, 105, 155; 2000, 113, 15. 


$$
\bar{M}_{i}(R, C)=\frac{M_{i}(R, C)-\left\langle M_{i}(R, C)\right\rangle}{\left\langle M_{i}(R, C)\right\rangle}
$$

where the brackets denote average over all $R$ and $C$ values. The correlations $C_{i, j}$ are calculated using the normalized mean-squareerror matrics $\bar{M}_{i}(R, C)$ as

$$
C_{i, j}=\frac{\left\langle\bar{M}_{i}(R, C) \bar{M}_{j}(R, C)\right\rangle}{\sqrt{\left\langle\bar{M}_{i}(R, C) \bar{M}_{i}(R, C)\right\rangle} \sqrt{\left\langle\bar{M}_{j}(R, C) \bar{M}_{j}(R, C)\right\rangle}}
$$

Correlations calculated this way may give nonzero $C_{i, j}$ for binding energies having very low intensity. In order to enhance contrast of the 2-D correlation plot, for each binding energy pair $i$ and $j$, the correlation value $C_{i, j}$ is multiplied by the peak intensities $S_{i} \times S_{j}$ of the grounded XPS data, giving $D_{i, j}=C_{i, j} S_{i} S_{j}$. The resulting matrix $D_{i, j}$ can be plotted as a contour plot or as a colorscale image for further inspection and interpretation. The correlations are, in effect, correlations of how the signal at particular binding energies increase or decrease as a function of frequency. The method works best for well separated peaks originating from one type of dynamic behavior. However, the strength of the method is that even for overlapping peaks with multiple time constants, correlations can be observed without fits to the data.

The method applied to the XPS data gives the two-dimensional correlation plot displayed in Figure 5 for the same $\mathrm{Ge}(\mathrm{np}) / \mathrm{GeO}_{\mathrm{y}} /$ $\mathrm{SiO}_{\mathrm{x}} / \mathrm{Si}$ composite sample. Naturally, all peaks display high autocorrelations given on the diagonal. However, the detailed and very rich information is in the cross-correlations (off-diagonals) between various peaks. For example, the $\mathrm{Si}^{0}$ and $\mathrm{Ge}^{0}(\mathrm{np})$ and also the $\mathrm{SiO}_{\mathrm{x}}, \mathrm{GeO}_{\mathrm{y}}$, and $\mathrm{O} 2 \mathrm{~s}$ peaks display strong cross-correlations as expected, but the distinction between the two oxides is also evident (i.e., the oxide around $\mathrm{Ge}$ and silicon oxide have different correlations, hence different dielectric properties). Moreover,

(16) (a) Curtis, O. L., Jr.; Srour, J. R. J. Appl. Phys. 1977, 48, 3819. (b) Hughes, R. C. Phys. Rev. B 1977, 15, 2012. (c) Wang, T.; Cheng, T. E.; Chiang, L. P.; Wang, C. H.; Zons, N. K.; Huang, C. IEEE Trans. Electron Devices 1998, 45, 1511.

(17) Kang, C. J.; Bush, G. H.; Lee, S.; Kim, C. K.; Mang, K. M.; Im, C.; Kuk, Y. Appl. Phys. Lett. 1989, 54, 338. closer examination of the cross-correlation plots reveal the presence of several hidden peaks, three of which correlate with the $\mathrm{Si}^{0}$ and four with $\mathrm{Si}^{4+}$, the nature of which will be examined and reported later. Note that this kind of information can not obtained using the conventional peak-fitting procedures, which always require certain (educated) guess-work.

We must also point out that the technique needs a lot of care in interpreting all of the correlation features, since some of them may be artifacts of the computational methodology introduced. These and other related issues like the advantages and/or disadvantages of this technique when compared with others like Fourier transform, etc. will be the subject of our future work.

In summary, we have developed a technique for obtaining twodimensional X-ray photoelectron spectroscopic data derived from the frequency dependence of the peaks recorded under electrical square-wave stimuli, which controls and affects the binding energy positions via the electrical potentials developed as a result of charging. By using cross-correlations between various peaks, our technique enables us to elucidate electrical characteristics of surface structures of composite samples. The approach is simple, versatile, and most importantly a noncontact measurement technique, which we expect to be most useful for investigation of fragile organic or biochemical layers, where conventional electrical measurements are difficult to perform. The resolution (the widths) of XPS peaks are inherently poorer compared with NMR peaks, making it more difficult to fully exploit the numerous analytical advantages of the latter technique. However, there is obviously still a lot of room for improvising like using different forms of stimuli (sine, triangular, etc.). Finally, although, we have utilized only electrical stimuli, extension to other forms of stimuli (optical, chemical, thermal, mechanical, etc.) can easily be realized.

\section{ACKNOWLEDGMENT}

This work was partially supported by TUBA (Turkish Academy of Sciences) and TUBITAK (The Scientific and Technological Research Council of Turkey) through the Grant No. 106T409.

Received for review December 31, 2007. Accepted April 2, 2008.

AC702642W 